\title{
Effect of the Phase Volume Ratio on the Potential of a Liquid-Membrane Ion-Selective Electrode
}

\author{
Zdeněk Samec*,t and Hubert H. Girault' \\ J. Heyrovsky Institute of Physical Chemistry, Academy of Sciences of the Czech Republic, Dolejškova 3, \\ 18223 Prague 8, Czech Republic, and Laboratoire d'Electrochimie Physique et Analytique, Institut de Chimie Moleculaire et \\ Biologique, Ecole Polytechnique Fédérale de Lausanne, CH-1015 Lausanne, Switzerland
}

Two-phase liquid system $I A(w) \mid I X(0)$ comprising the interface between the aqueous solution $(w)$ of uni-univalent electrolyte IA and an organic solvent solution (o) of a uni-univalent electrolyte IX with the common cation $\mathrm{I}^{+}$is considered as a simple model of a liquid-membrane ion-selective electrode (ISE). Taking into account the electroneutrality and mass balance conditions, the equilibrium Galvani potential difference (pd) between the aqueous and organic phases, $\Delta_{0}^{\mathrm{w}} \phi=\phi(w)-\phi(0)$, is calculated numerically as a function of the ratio of the initial electrolyte concentrations, $x=c_{I A}^{0} / c_{I X}^{0}=10^{-4}$ $10^{4}$, for the selected values of the phase volume ratio $r=$ $\mathrm{V}(\mathrm{o}) / \mathrm{V}(\mathrm{w})=10^{-3}, 1$, and $10^{3}$, and the standard ion transfer potentials of the present ions ranging from -0.5 to $0.5 \mathrm{~V}$. Numeric results corroborate the symbolic expressions derived for the cases when $\mathrm{X}^{-}$and $\mathrm{A}^{-}$are extremely lipophilic and hydrophilic ions, respectively, or when the concentration ratio $x$ is extremely large or small. In contrast to the extraction system, where both electrolytes are initially present in the aqueous phase, the effect of the phase volume ratio on the equilibrium pd in the ISE model is rather weak, unless the counterions $X^{-}$and $A^{-}$differ little in their lipophilicity from the target ion $\mathrm{I}^{+}$. It is shown that both the ISE and extraction model exhibit the Nernstian behavior only in a limited range of the concentration ratio $x$ depending on the value of the standard ion transfer potentials of the counterions. When this ratio is extremely large or small, equilibrium pd approaches the limiting value given by the distribution potential of the electrolyte IA or IX, respectively. Similar conclusions can be drawn for the two-phase liquid system $\mathrm{Al}(\mathbf{w}) \mid \mathrm{XI}(0)$ with the common anion $\mathrm{I}^{-}$.

Liquid-membrane ion-selective electrodes (ISE) are heterogeneous electrochemical systems consisting of a plastic film (e.g., poly(vinyl chloride)), whose matrix contains an ion-exchanger solution as a plasticizer. ${ }^{1}$ From the point of view of electrochemistry at the interface between two immiscible electrolyte solutions $\left(\right.$ ITIES), ${ }^{2}$ they represent the multi-ion partition systems, which are characterized by the prevailing contribution of one ion to the

\footnotetext{
* Corresponding author. Tel.: +420-266052017. Fax: +420-286582307. Email: zdenek.samec@jh-inst.cas.cz.

+ Academy of Sciences of the Czech Republic.

‡ Ecole Polytechnique Fédérale de Lausanne.
}

\section{Scheme 1}

$$
\text { IA, } \mathrm{H}_{2} \mathrm{O} \mid \mathrm{IX}, \mathrm{S}
$$

ISE potential. The simplest ITIES model of the liquid-membrane ISE comprises two uni-univalent electrolytes IX and IA with the common cation $\mathrm{I}^{+}$, which are separated by the interface between the aqueous ( $w$ ) and the organic solvent phase (o) (Scheme 1), where $\mathrm{S}$ represents an organic solvent. The general method to calculate the equilibrium Galvani potential difference $\Delta_{0}^{\mathrm{w}} \phi=$ $\phi(\mathrm{w})-\phi(0)$ for such a two-phase liquid system was developed on the basis of the mass balance equations and the electroneutrality condition for each phase, which were combined with the Nernst equations for all the present ions. ${ }^{3}$ The application of this method to various systems indicated that the equilibrium and initial concentrations of ions commonly differ. ${ }^{3}$ Hence, Scheme 1 describes rather the initial than the equilibrium state, with the initial ion concentrations ${ }^{i} c_{k}(w)$ and ${ }^{i} c_{k}(0)$ in the phase $w$ and 0 , respectively, given by

$$
\begin{gathered}
{ }^{i} c_{1+}(w)={ }^{i} c_{A-}(w)=c_{1 A}^{0} \\
{ }^{i} c_{X-}(w)=0 \\
{ }^{i} c_{1+}(0)={ }^{i} c_{X-}(0)=c_{1 X}^{0} \\
{ }^{i} c_{A^{-}}(0)=0
\end{gathered}
$$

where $c_{1 A}^{0}$ and $c_{I X}^{0}$ are the analytical concentrations of the elec-

(1) (a) M orf, W. E. In Studies in Analytical Chemistry 2: The Principles of I onSelective Electrodes and of Membrane Transport; Pungor, E., Simon, W., Inczédy, J., Eds.; Akadémiai Kiadó: Budapest, 1981. (b) Koryta, J.; ×b3tulík, K. In I on-Selective Electrodes, 2nd ed.; Cambridge University Press: London, 1983. (c) Bakker, E.; Bühlmann, P.; Pretsch, E. Electroanalysis 1999, 11, 915-933.

(2) (a) Girault, H. H. J.; Schiffrin, D. J. In Electroanalytical Chemistry; Bard, A. J., Ed.; M arcel Dekker: New York, 1989; Vol. 15, pp 1-141. (b) Girault, H. H. In Modern Aspects of Electrochemistry; Bockris, J. O'M., Ed.; Plenum Press: New York, 1983; Vol. 25, pp 1-62. (c) Samec, Z.; Kakiuchi, T. In Advances in Electrochemistry and Electrochemical Science; Alkire, R. C. Gerischer, H., Kolb, D. M., Tobias, C. W., Eds.; Wiley-VCH: Weinheim, 1995; Vol. 4, pp 297-361. (d) Liquid-Liquid Interfaces: Theory and M ethods; Volkov, A. G., Deamer, D. W., Eds.; CRC Press: Boca Raton, FL, 1996. (f) Reymond, F.; Fermín, D.; Lee, H. J.; Girault, H. H. Electrochim. Acta 2000 45, 2647-2662.

(3) Hung, L. Q. J. Electroanal. Chem. 1980, 115, 159-174. 


\section{Scheme 2}

IA, IX, $\mathrm{H}_{2} \mathrm{O} \mid \mathrm{S}$

(w)

(o)

trolytes IA and IX, respectively. However, if X- is a highly lipophilic ion and $\mathrm{A}^{-}$is a highly hydrophilic ion, the equilibrium and initial concentrations of the $\mathrm{I}^{+}$ion can be equal, and the potential difference $\Delta_{0}^{\mathrm{w}} \phi$ is controlled by the ratio of activities or concentrations of $\mathrm{I}^{+}$, which then becomes the potential-determining ion. ${ }^{3}$

Another aspect of the ion partition equilibrium in the two-phase liquid system is the effect of the ratio $r$ of the volumes $V(0)$ and $\mathrm{V}(\mathrm{w})$ of the organic and the aqueous phase, respectively, that is,

$$
r=\frac{V(0)}{V(w)}
$$

on the equilibrium potential difference and ion concentrations, which is introduced by virtue of the mass balance equation. ${ }^{3}$ The role of the phase volume ratio has not been fully recognized until recently when the limiting behavior in the equilibrium ion partition between two phases of considerably different volumes has been analyzed, and the strong effect of the phase volume ratio on the equilibrium potential difference $\Delta_{0}^{\mathrm{w}} \phi$ has been demonstrated for the extraction system (Scheme 2) where the electrolytes IA and $I X$ are initially present in the aqueous phase; that is, the initial ion concentrations are given by the equations ${ }^{4}$

$$
\begin{gathered}
{ }^{\mathrm{i}} \mathrm{c}_{1+}(\mathrm{w})=\mathrm{c}_{\mathrm{IA}}^{0}+\mathrm{c}_{\mathrm{IX}}^{0} \\
{ }^{\mathrm{i}} \mathrm{c}_{\mathrm{A}}(\mathrm{w})=\mathrm{c}_{\mathrm{IA}}^{0} \\
{ }^{\mathrm{i}} \mathrm{c}_{\mathrm{X}-}(\mathrm{w})=\mathrm{c}_{\mathrm{IX}}^{0} \\
{ }^{\mathrm{i}} \mathrm{c}_{1+}(0)={ }^{\mathrm{i}} \mathrm{c}_{\mathrm{X}-}(0)={ }^{\mathrm{i}} \mathrm{c}_{\mathrm{A}^{-}}(0)=0
\end{gathered}
$$

Such analysis can be relevant to theoretical treatments of the separation (extraction) processes or the ion transport processes taking place at biological membranes. ${ }^{4}$ It is to be noted that this type of two-phase system is also encountered in the voltammetric measurements of the ion transfer reactions on thin liquid films ${ }^{5}$ or liquid droplets ${ }^{6-9}$ supported on a solid electrode.

The main aim of this work was to analyze the effect of the phase volume ratio on the equilibrium potential difference $\Delta_{0}^{\mathrm{w}} \phi$ for the ITIES model of the liquid-membrane ISE described by Scheme 1 , with the initial conditions given by eq 1 . We shall show

(4) Kakiuchi, T. Anal. Chem. 1996, 68, 3658-3664.

(5) (a) Shi, C.; Anson, F. C. J. Phys. Chem. B 1998, 102, 9850-9854. (b) Shi, C.; Anson, F. C. Anal. Chem. 1998, 70, 3114-3118. (c) Shi, C.; Anson, F. C. J. Phys. Chem. B 1999, 103, 6283-6289.

(6) (a) Scholz, F.; Komorsky-Lovric, S.; Lovric, M. Electrochem. Commun. 2000, 2, 112-118. (b) Komorsky-Lovric, S.; Lovric, M.; Scholz, F. J. Electroanal. Chem. 2001, 508, 129-137.

(7) Ulmeanu, S.; Lee, H. J .; Fermin, D.; Girault, H. H.; Shao, Y. H. Electrochem. Commun. 2001, 3, 219-223.

(8) Zhang, M.; Sun, P.; Chen, Y.; Li, F.; Gao, Z.; Shao, Y. Anal. Chem. 2003, 75, 4341-4345.

(9) (a) Tasakorn, P.; Chen, J.; Aoki, K. J. Electroanal. Chem. 2002, 533, 119126. (b) Aoki, K.; Tasakorn, P.; Chen, J. J. Electroanal. Chem. 2003, 542, $51-60$.

\section{Scheme 3}

AI, $\mathrm{H}_{2} \mathrm{O} \mid \mathrm{XI}, \mathrm{S}$

(w)

(o)

that unless the counterions $\mathrm{X}^{-}$and $\mathrm{A}^{-}$differ little in their lipophilicity from the target ion $\mathrm{I}^{+}$, this effect is rather weak, in contrast to that predicted ${ }^{4}$ for the extraction system, Scheme 2. For both ISE and the extraction system, we shall also examine in a detail the dependence of $\Delta_{0}^{\mathrm{w}} \phi$ on the ratio of the electrolyte concentrations $x$

$$
x=\frac{c_{1 A}^{0}}{c_{I X}^{0}}
$$

for a fixed value of the phase volume ratio $r$. We shall demonstrate that, depending on the lipophilicity of the counterions $\mathrm{X}^{-}$and $\mathrm{A}^{-}$, the Nernstian behavior can be observed only in a range of the concentration ratio $x$. Expressions for the equilibrium potential difference $\Delta_{0}^{\mathrm{w}} \phi$ when this ratio is extremely large or small will be derived. The analysis will be extended to the interface between the aqueous solution of uni-univalent electrolyte $\mathrm{Al}$ and an organic solvent solution of a uni-univalent electrolyte XI with the common anion $\mathrm{I}^{-}$(Scheme 3).

\section{MODEL EQUATIONS}

Following the general treatment, ${ }^{3}$ the equilibrium potential difference $\Delta_{0}^{\mathrm{w}} \phi$ for the system described by Scheme 1 can be calculated by combining the electroneutrality condition for, for example, the aqueous phase,

$$
c_{1+}(w)-c_{X-}(w)-c_{A-}(w)=0
$$

with the mass balance and the Nernst equations, respectively,

$$
\begin{gathered}
c_{k}(w)+r c_{k}(0)=\frac{n_{k}^{\text {tot }}}{V(w)}=\xi_{k} \\
\Delta_{0}^{w} \phi=\Delta_{0}^{w} \phi_{k}^{0}+\frac{R T}{z_{k} F} \ln \frac{a_{k}(0)}{a_{k}(w)}=\Delta_{0}^{w} \phi_{k}^{0^{\prime}}+\frac{R T}{z_{k} F} \ln \frac{c_{k}(0)}{c_{k}(w)}
\end{gathered}
$$

for the ions $\mathrm{k}=\mathrm{I}^{+}, \mathrm{X}^{-}$, or $\mathrm{A}^{-}$, where $\mathrm{z}_{\mathrm{k}}$ is the ion charge number, $a_{k}$ 's are the ion activities, $c_{k}$ 's are the equilibrium ion concentrations, and $\Delta_{0}^{\mathrm{w}} \phi_{\mathrm{k}}^{0}$ is the standard ion transfer potential, which is given by the standard Gibbs energy of ion transfer from the aqueous phase to the organic phase, $\Delta \mathrm{G}_{\mathrm{K}}^{0, w \rightarrow 0}$,

$$
\Delta_{0}^{\mathrm{w}} \phi_{\mathrm{k}}^{0}=\frac{\Delta \mathrm{G}_{\mathrm{k}}^{0, \mathrm{w} \rightarrow 0}}{\mathrm{z}_{\mathrm{k}} \mathrm{F}}
$$

and $\Delta_{0}^{\mathrm{w}} \phi_{\mathrm{k}}^{0^{\prime}}$ is the formal ion transfer potential including the ion activity coefficients. The value of $\Delta \mathrm{G}_{\mathrm{k}}^{0, w \rightarrow 0}$ is a measure of the ion lipophilicity; that is, an ion is considered as lipophilic or hydrophilic when this value is negative or positive, respectively. Equation 6 defines the parameter $\xi_{\mathrm{k}}$ as the total number of moles 
$n_{k}^{\text {tot }}$ of the ion $k$ in the system related to the volume of the aqueous phase $\mathrm{V}(\mathrm{w})$. There is a significant difference between the parameter $\xi_{\mathrm{k}}$ for the system where the initial concentrations are given by eq 1 and by eq 3 . In the former case, $\xi_{I^{+}}=c_{I A}^{0}+$ $r c_{I X}^{0}, \xi_{X^{-}}=r c_{I X}^{0}$, and $\xi_{A^{-}}=c_{I A}^{0}$, while in the latter case, $\xi_{I^{+}}=c_{I A}^{0}+$ $c_{I X}^{0}, \xi_{X^{-}}=c_{I X}^{0}$, and $\xi_{A^{-}}=c_{I A}^{0}$. Equation 5 can be then rewritten as

$$
\begin{aligned}
& \frac{\xi_{1+}}{1+r \exp \left[\mathrm{F}\left(\Delta_{0}^{\mathrm{w}} \phi-\Delta_{0}^{\mathrm{w}} \phi_{I^{\prime}}^{\mathrm{o}^{\prime}}\right) / \mathrm{RT}\right]}- \\
& \frac{\xi_{\mathrm{X}-}}{1+\mathrm{r} \exp \left[-\mathrm{F}\left(\Delta_{0}^{\mathrm{w}} \phi-\Delta_{0}^{\mathrm{w}} \phi_{\mathrm{X}^{-}}{ }^{0^{\prime}}\right) / \mathrm{RT}\right]}- \\
& \frac{\xi_{\mathrm{A}^{-}}}{1+\mathrm{r} \exp \left[-\mathrm{F}\left(\Delta_{0}^{\mathrm{w}} \phi-\Delta_{0}^{\mathrm{w}} \phi_{\mathrm{A}^{-}}{ }^{0^{\prime}}\right) / \mathrm{RT}\right]}=0
\end{aligned}
$$

which makes it possible to calculate $\Delta_{0}^{\mathrm{w}} \phi$ as a function of the concentration ratio $x$ given by eq 4 , for the particular values of the phase volume ratio $r$ and the formal ion transfer potentials $\Delta_{0}^{\mathrm{w}} \phi_{\mathrm{k}}^{0^{\prime \prime} \mathrm{s}}$. For this purpose, it is convenient to express eq 9 in the dimensionless form, which for the ITIES model with the initial concentrations given by eq 1 becomes

$$
y=\frac{r+x}{1+r \beta_{I^{+}}{ }^{-1} s}-\frac{r}{1+r \beta_{x_{-}} s^{-1}}-\frac{x}{1+r \beta_{A_{-}-s^{-1}}}=0
$$

and for the ITIES model with the initial concentrations given by eq 3 becomes

$$
y=\frac{1+x}{1+r \beta_{I^{+}}{ }^{-1} s^{-}}-\frac{1}{1+r \beta_{X_{-}-s^{-1}}}-\frac{x}{1+r \beta_{A_{-}-s^{-1}}}=0
$$

where

$$
\begin{aligned}
\mathrm{s} & =\exp \left[\mathrm{F} \Delta_{\mathrm{o}}^{\mathrm{w}} \phi / \mathrm{RT}\right] \\
\beta_{\mathrm{k}} & =\exp \left[\mathrm{F} \Delta_{0}^{\mathrm{w}} \phi_{\mathrm{k}}^{0^{\prime}} / \mathrm{RT}\right]
\end{aligned}
$$

Extension of this treatment to the system with the common anion $\mathrm{I}^{-}$described by Scheme 3 is straightforward. In this case, the electroneutrality condition reads

$$
\mathrm{c}_{\mathrm{A}^{+}}(\mathrm{w})+\mathrm{c}_{\mathrm{X}+}(\mathrm{w})-\mathrm{c}_{-}(\mathrm{w})=0
$$

Equilibrium potential difference $\Delta_{0}^{\mathrm{w}} \phi$ can again be calculated by using eq 10 or eq 11, where

$$
x=\frac{C_{A I}^{0}}{C_{X I}^{0}}
$$

and the parameters $\mathrm{s}$ and $\beta_{\mathrm{k}}$ are replaced by new parameters $\mathrm{p}$ and $\gamma_{k}$, respectively,

$$
\begin{aligned}
p & =\exp \left[-F \Delta_{0}^{w} \phi / R T\right] \\
\gamma_{k} & =\exp \left[-F \Delta_{0}^{w} \phi_{k}^{0^{\prime}} / R T\right]
\end{aligned}
$$

\section{SYMBOLIC SOLUTION}

To calculate the equilibrium potential difference $\Delta_{0}^{\mathrm{w}} \phi$, eqs 10 and 11 have to be solved numerically, but symbolic expressions can be derived, which are valid in a range of the parameters involved. Since the limiting cases when the phase volume ratio $r$ is either extremely small or large has been analyzed previously for one value of $x=10^{5}, 4$ we shall focus on the limiting cases associated with the concentration ratio $x$, which are rather of analytical interest.

First, to illustrate the difference between the ISE and extraction ITIES models with the common cation $\mathrm{I}^{+}$, we shall assume that $\mathrm{X}^{-}$and $\mathrm{A}^{-}$are extremely lipophilic and hydrophilic ions, that is, their standard ion transfer potentials $\Delta_{0}^{\mathrm{w}} \phi_{\mathrm{X}^{-}}^{0} \rightarrow \infty$ and $\Delta_{0}^{\mathrm{w}} \phi_{\mathrm{A}^{-}}^{0} \rightarrow$ $-\infty$, respectively. These limits actually correspond to the condition that ions $X^{-}$and $A^{-}$are bound to the corresponding phases. Since then $r \beta_{X^{-}} S^{-1} \gg 1$ and $r \beta_{A}-S^{-1} \ll 1$ for any finite value of the product $\mathrm{rs}^{-1}$, the second term on the right-hand side of eqs 10 and 11 can be neglected and two symbolic expressions follow, respectively:

$$
\begin{gathered}
\Delta_{0}^{\mathrm{w}} \phi=\frac{\mathrm{RT}}{\mathrm{F}} \ln \frac{\beta_{I^{+}}}{\mathrm{x}}=\Delta_{0}^{\mathrm{w}} \phi_{I^{\prime}}^{0^{\prime}}+\frac{\mathrm{RT}}{\mathrm{F}} \ln \frac{\mathrm{C}_{\mathrm{IX}}^{0}}{\mathrm{C}_{\mathrm{IA}}^{0}} \\
\Delta_{0}^{\mathrm{w}} \phi=\frac{\mathrm{RT}}{\mathrm{F}} \ln \frac{\beta_{I^{+}}}{\mathrm{rX}}=\Delta_{0}^{\mathrm{w}} \phi_{I^{\prime}}^{0^{\prime}}+\frac{\mathrm{RT}}{\mathrm{F}} \ln \frac{\mathrm{C}_{\mathrm{IX}}^{0}}{\mathrm{C}_{\mathrm{IA}}^{0}}-\frac{\mathrm{RT}}{\mathrm{F}} \ln \mathrm{r}
\end{gathered}
$$

Equation 18, which has been obtained previously in an analogous way, ${ }^{3}$ shows that the equilibrium potential difference $\Delta_{0}^{\mathrm{w}} \phi$ is the logarithmic function of the ratio of the initial concentrations of the ion $\mathrm{I}^{+}, \mathrm{C}_{\mathrm{IX}}^{0} / \mathrm{C}_{\mathrm{IA}}^{0}={ }^{\mathrm{i}} \mathrm{C}_{\mathrm{I}^{+}}(0) /{ }^{i} \mathrm{C}_{1}{ }^{+}(\mathrm{w}), \mathrm{cf}$. eq 1 , but it is independent of the phase volume ratio $r$. On the other hand, the potential difference for the extraction model is predicted to exhibit the logarithmic dependence on the phase volume ratio $r$, in addition to the logarithmic dependence on the ratio of the initial concentrations of the counterions in the extracted (aqueous) phase $c_{I X}^{0} / c_{I A}^{0}$ $={ }^{i} C_{X}-(w) /{ }^{i} C_{A}-(w), c f$. eq 3 . The effect of the phase volume ratio thus appears to be a consequence of the initial conditions, assuming the zero initial concentrations of the partitioned ions in the extracting (organic) phase. The logarithmic dependence on $r$ was first demonstrated by numeric calculation, ${ }^{4}$ but the explicit expression, eq 19 , has not been introduced until more recently in voltammetric studies of the effect of phase volume ratio on the ion transfer. ${ }^{10}$

Symbolic expressions that apply to both the ISE and extraction model can be obtained for the large and small values of the ratio of the electrolyte concentrations $\mathrm{X}$, provided that $\Delta_{0}^{\mathrm{w}} \phi_{\mathrm{A}^{-}}^{0} \ll \Delta_{0}^{\mathrm{w}} \phi_{1^{+}}^{0}$ $\ll \Delta_{0}^{\mathrm{w}} \phi_{\mathrm{X}-}^{0}$ or $\Delta_{0}^{\mathrm{w}} \phi_{\mathrm{X}^{+}}^{0} \ll \Delta_{0}^{\mathrm{w}} \phi_{\mathrm{I}^{-}}^{0} \ll \Delta_{0}^{\mathrm{w}} \phi_{\mathrm{A}^{+}}^{0}$. When $\mathrm{x} \gg 1$, the potential difference $\Delta_{0}^{w} \phi$ tends to acquire large and negative or positive value, respectively, cf., for example, eqs 18 and 19. An estimate of the terms appearing on the right-hand side of eqs 10 and 11 suggests that the second term can be neglected, and the following expressions are obtained,

$$
\Delta_{0}^{\mathrm{w}} \phi(\mathrm{x} \gg 1)=\frac{\mathrm{RT}}{\mathrm{F}} \ln \sqrt{\beta_{I_{+}} \beta_{\mathrm{A}^{-}}}=\frac{\Delta_{0}^{\mathrm{w}} \phi_{1^{\prime}}^{0^{\prime}}+\Delta_{0}^{\mathrm{w}} \phi_{\mathrm{A}^{-}}^{0^{\prime}}}{2}
$$

or

$$
\Delta_{0}^{\mathrm{w}} \phi(x \gg 1)=-\frac{\mathrm{RT}}{\mathrm{F}} \ln \sqrt{\gamma_{\mathrm{A}^{+}} \gamma_{\mathrm{I}^{-}}}=\frac{\Delta_{0}^{\mathrm{w}} \phi_{\mathrm{A}^{+}}^{0^{0^{\prime}}}+\Delta_{0}^{\mathrm{w}} \phi_{1^{0^{\prime}}}^{0^{\prime}}}{2}
$$



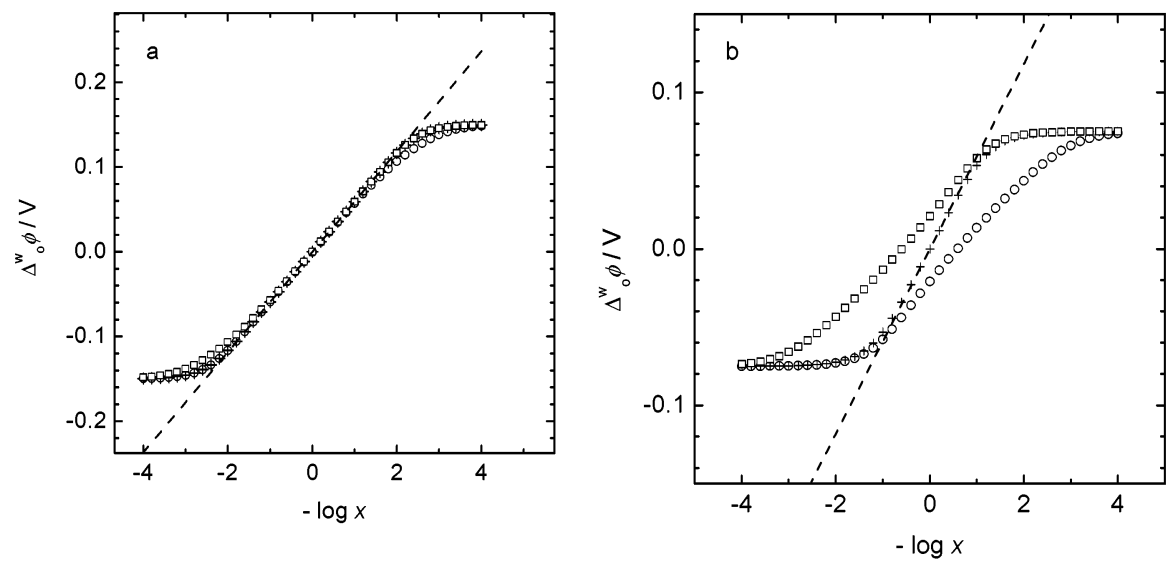

Figure 1. Equilibrium potential difference $\Delta_{0}^{\mathrm{w}} \phi$ for the ITIES model of the liquid-membrane ISE vs the logarithm of the concentration ratio $x=$ $c_{\mathrm{IA}}^{0} / c_{\mathrm{IX}}^{0}$ calculated by using eq 10 for the phase volume ratio $r=10^{-3}(O), 1(+)$, and $10^{3}(\square)$ and two different sets of values of the standard ion transfer potentials: (a) $\Delta_{0}^{\mathrm{w}} \phi_{1^{+}}^{0}=0, \Delta_{\mathrm{o}}^{\mathrm{w}} \phi_{\mathrm{A}^{-}}^{0}=-0.3 \mathrm{~V}$, and $\Delta_{0}^{\mathrm{w}} \phi_{\mathrm{X}^{-}}^{0}=0.3 \mathrm{~V}$ or (b) $\Delta_{0}^{\mathrm{w}} \phi_{1^{+}}^{0}=0, \Delta_{0}^{\mathrm{w}} \phi_{\mathrm{A}^{-}}^{0}=-0.15 \mathrm{~V}$, and $\Delta_{0}^{\mathrm{w}} \phi_{\mathrm{X}^{-}}^{0}=0.15 \mathrm{~V}$. Dashed line corresponds to the ideal Nernstian behavior according to eq 17.

for the system with the common cation or anion, respectively. In this case, the potential difference is given by the distribution potential11 of the electrolyte IA or AI irrespective of the phase volume ratio $r$. The same expressions were derived in the other way as the limit for a large extreme of $r$ and a constant and large value of $x^{4}$ On the other hand, when $x \ll 1$, the third term on the right-hand side of eqs 10 and 11 becomes rather negligible, and the equilibrium potential difference approaches the distribution potential of the electrolyte IX or XI, respectively,

$$
\Delta_{0}^{\mathrm{w}} \phi(\mathrm{x} \ll 1)=\frac{\mathrm{RT}}{\mathrm{F}} \ln \sqrt{\beta_{1+} \beta_{\mathrm{X}-}}=\frac{\Delta_{0}^{\mathrm{w}} \phi_{1^{+}}^{0^{\prime}}+\Delta_{0}^{\mathrm{w}} \phi_{\mathrm{X}-}^{0^{\prime}}}{2}
$$

or

$$
\Delta_{0}^{\mathrm{w}} \phi(x \ll 1)=-\frac{\mathrm{RT}}{\mathrm{F}} \ln \sqrt{\gamma_{\mathrm{X}+} \gamma_{1^{-}}}=\frac{\Delta_{0}^{\mathrm{w}} \phi_{\mathrm{X}+}^{0^{\prime}}+\Delta_{0}^{\mathrm{w}} \phi_{1^{\prime}}^{0^{\prime}}}{2}
$$

for the system with the common cation or anion, respectively. Thus, irrespective of the initial conditions and the phase volume ratio, the limiting value of the potential difference is given by the distribution potential of the electrolyte that is present in excess.

Both the ISE and the extraction model exhibits a change from the Nernstian to non-N ernstian behavior at a value of the ratio of electrolyte concentration, which for the system with the common cation $\mathrm{I}^{+}$can be obtained by substitution of eqs 20 and 22 into eqs 18 or 19. For the ISE model, this change occurs when this ratio reaches the value as large as $x_{1}^{\lim }=\left(\beta_{1^{+}}+\beta_{A^{-}}\right)^{1 / 2}$ or as small as $x_{s}^{\lim }=\left(\beta_{1^{+}} / \beta_{X^{-}}\right)^{1 / 2}$. For the extraction model, these values depend on the phase volume ratio $r$, that is, $x_{1}^{\lim }=r^{-1}\left(\beta_{1^{+}} / \beta_{A^{-}}\right)^{1 / 2}$ and $x_{s}^{\lim }=r^{-1}\left(\beta_{I^{+}}+\beta_{X^{-}}\right)^{1 / 2}$. Similar expressions can be derived for the system with the common anion $\mathrm{I}^{-}$, for example, for the ISE model $x_{1}^{\lim }=\left(\gamma_{1}-/ \gamma_{A^{+}}\right)^{1 / 2}$ and $x_{s}^{\lim }=\left(\gamma_{1}-/ \gamma_{x^{+}}\right)^{1 / 2}$.

\section{NUMERIC CALCULATION}

Correctness of the symbolic expressions given above can be confirmed by numeric calculations for the system with the

(10) Gobry, V.; Ulmeanu, S.; Reymond, F.; Bouchard, G.; Carrupt, P.; Testa, B.; Girault, H. H. J. Am. Chem. Soc. 2001, 123, 10684-10690.

(11) Karpfen, F. M.; Randles, J. E. B. Trans. Faraday Soc. 1953, 49, 823-831.

\section{Table 1. Standard Ion Transfer Potentials Obtained from the Voltammetric Measurements of the Ion Transfer from Water to o-NPOE ${ }^{12,13}$ or the o-NPOE

\begin{tabular}{|c|c|c|c|c|c|c|c|}
\hline \multirow[b]{2}{*}{ iona $^{a}$} & \multicolumn{3}{|c|}{$\Delta_{0}^{\mathrm{w}} \phi_{\mathrm{i}} \% \mathrm{~V}$} & \multirow[b]{2}{*}{ ion $^{a}$} & \multicolumn{3}{|c|}{$\Delta_{0}^{\mathrm{w}} \phi_{\mathrm{i}} \% \mathrm{~V}$} \\
\hline & ref 12 & ref 13 & ref 14 & & ref 12 & ref 13 & ref 14 \\
\hline $\begin{array}{l}\mathrm{Li}^{+} \\
\mathrm{K}^{+} \\
\mathrm{TBA}^{+} \\
\text {TPAS }^{+}\end{array}$ & $\begin{array}{r}0.488 \\
-0.242 \\
-0.314\end{array}$ & $\begin{array}{r}0.340 \\
-0.316\end{array}$ & -0.290 & $\begin{array}{l}\mathrm{ClO}_{4}{ }^{-} \\
\mathrm{Cl}^{-} \\
\mathrm{TPB}^{-}\end{array}$ & $\begin{array}{r}-0.136 \\
0.314\end{array}$ & $\begin{array}{r}-0.175 \\
-0.521 \\
0.316\end{array}$ & 0.290 \\
\hline
\end{tabular} Plasticized PVC Membrane (Last Column) ${ }^{14}$}

a Abbreviations: tetrabutylammonium ${ }^{+}\left(\mathrm{TBA}^{+}\right)$, tetraphenylarsonium $^{+}\left(\mathrm{TPAs}^{+}\right)$, and tetraphenylborate $\left(\mathrm{TPB}^{-}\right)$.

common cation $\mathrm{I}^{+}$. By using the Mathcad Equation Solver ( $M$ athcad 8 Professional, $M$ athSoft, Inc.), the equilibrium potential difference $\Delta_{0}^{\mathrm{w}} \phi$ was calculated from eqs 10 and 11 as a function of the ratio of electrolyte concentrations $x=10^{-4}-10^{4}$, for several values of the phase volume ratio $r$ and of the parameters $\beta_{\mathrm{k}}=$ $\exp \left[F \Delta_{0}^{\mathrm{w}} \phi_{\mathrm{k}}^{0^{\prime}} / \mathrm{RT}\right]$. Since the volume of an ISE liquid membrane is typically around $100 \mu \mathrm{L}$ and the volume of the aqueous sample is usually $1-100 \mathrm{~mL}$, the phase volume ratio for an ISE is rather small and falls in the range $10^{-1}-10^{-3}$. Therefore, the potential difference was calculated for $x=10^{-3}$ and, for the purpose of comparison, also for $x=1$ and $10^{3}$. The values of the parameters $\beta_{\mathrm{k}}$ used in calculation were based on the standard ion transfer potentials $\Delta_{0}^{\mathrm{w}} \phi_{\mathrm{k}}^{0}$ evaluated from voltammetric measurements on water|0-nitrophenyl octyl ether (O-NPOE) interface ${ }^{12,13}$ and the O-NPOE plasticized PVC membrane. ${ }^{14}$ Table 1 summarizes the selected data, suggesting that $\Delta_{0}^{\mathrm{w}} \phi_{\mathrm{k}}^{0}$ attains the maximum value of ca. $\pm 0.5 \mathrm{~V}$. Therefore, the equilibrium potential difference was calculated for $\Delta_{0}^{\mathrm{w}} \phi_{1^{+}}^{0}=0 \mathrm{~V}, \Delta_{0}^{\mathrm{w}} \phi_{\mathrm{X}^{-}}^{0}=0.15,0.3,0.4$, or $0.5 \mathrm{~V}$ and $\Delta_{0}^{\mathrm{w}} \phi_{\mathrm{A}^{-}}^{0}=-0.15,-0.3,-0.4$, or $-0.5 \mathrm{~V}$.

Figure 1 shows the effect of the phase volume ratio on the equilibrium potential difference $\Delta_{0}^{\mathrm{w}} \phi$ for the ITIES model of the

(12) Samec, Z.; Langmaier, J.; Trojánek, A. J. Electroanal. Chem. 1996, 409, 1-7.

(13) Wilke, S.; Zerihun, T. J. Electroanal. Chem. 2001, 515, 52-60.

(14) Langmaier, J.; Stejskalová, K.; Samec, Z. J. Electroanal. Chem. 2001, 496, 143-147. 


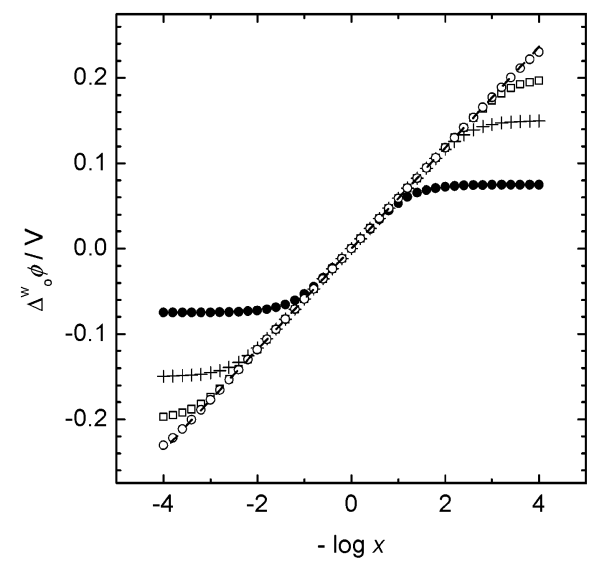

Figure 2. Equilibrium potential difference $\Delta_{\circ}^{\mathrm{w}} \phi$ for the ITIES model of the liquid-membrane ISE vs the logarithm of the concentration ratio $x=c_{\mathrm{IA}}^{0} / c_{\mathrm{IX}}^{0}$ calculated by using eq 10 for the phase volume ratio $r=$ $1, \Delta_{0}^{\mathrm{w}} \phi_{I^{+}}^{0}=0$, and the following values of the standard ion transfer potentials of the anions $\mathrm{A}^{-}$and $\mathrm{X}^{-}$, respectively: $\Delta_{0}^{\mathrm{w}} \phi_{\mathrm{A}^{-}}^{0}=-0.15 \mathrm{~V}$, $\Delta_{0}^{\mathrm{w}} \phi_{\mathrm{X}^{-}}^{0}=0.15 \mathrm{~V}(\bullet), \Delta_{\mathrm{o}}^{\mathrm{w}} \phi_{\mathrm{A}^{-}}^{0}=-0.3 \mathrm{~V}, \Delta_{\mathrm{o}}^{\mathrm{w}} \phi_{\mathrm{X}^{-}}^{0}=0.3 \mathrm{~V}(+), \Delta_{\mathrm{o}}^{\mathrm{w}} \phi_{\mathrm{A}^{-}}^{0}=$ $-0.4 \mathrm{~V}, \Delta_{\circ}^{\mathrm{w}} \phi_{\mathrm{X}^{-}}^{0}=0.4 \mathrm{~V}(\square)$, and $\Delta_{\mathrm{o}}^{\mathrm{w}} \phi_{\mathrm{A}^{-}}^{0}=-0.5 \mathrm{~V}, \Delta_{\mathrm{o}}^{\mathrm{w}} \phi_{\mathrm{X}^{-}}^{0}=0.5 \mathrm{~V}(\mathrm{O})$. Dashed line corresponds to the ideal Nernstian behavior according to eq 17.

liquid-membrane ISE plotted against the logarithm of the concentration ratio $\mathrm{x}=\mathrm{c}_{\mathrm{IA}}^{0} / \mathrm{c}_{\mathrm{IX}}^{0}$, assuming a larger (Figure la) or smaller (Figure 1 b) difference in the lipophilicity of the anions $\mathrm{X}^{-}$and $\mathrm{A}^{-}$from the target ion $\mathrm{I}^{+}$. In the former case, a weak effect of the phase volume ratio $r$ can be seen in the range of the concentration ratio, where the transition from the Nernstian to non-Nernstian behavior occurs. In the latter case, this effect is much more pronounced and, eventually, the Nernstian slope of $59 \mathrm{mV}$ is not to be expected in the whole range of the electrolyte concentration ratio, when the phase volume ratio differs from unity, cf. empty circles and squares. Figure 1 also illustrates the limiting behavior of the ISE model for the extreme values of the concentration ratio $x$. The effect of the standard ion transfer potential of the anions $X^{-}$and $A^{-}$on the limiting behavior at $x \gg$ 1 and $x \ll 1$ is shown in Figure 2 . In agreement with the symbolic solution, eq 20, the potential difference $\Delta_{0}^{\mathrm{w}} \phi$ approaches the value of $0.075,0.15,0.2$, or 0.25 at $x \ll 1$, when the standard ion transfer potential $\Delta_{0}^{\mathrm{w}} \phi_{\mathrm{x}-}^{0}=0.15,0.3,0.4$, or $0.5 \mathrm{~V}$, respectively. The inverse limit at $x \gg 1$ is analogously given by the selected values of the standard potential $\Delta_{0}^{\mathrm{w}} \phi_{\mathrm{A}}^{0}$; the limiting values from numeric calculations agree with those calculated from eq 22.

With use of the data given in Table 1 , the behavior of two practical systems has been examined, namely, that of TBACIITBATPB with the common $\mathrm{TBA}^{+}$cation, and $\mathrm{LiClO}_{4} \mid \mathrm{TBAClO}_{4}$ with the common $\mathrm{ClO}_{4}^{-}$anion. The results of calculations are shown in Figure 3. The model TBA ${ }^{+}$ion-selective electrode is predicted to exhibit the Nernstian behavior for the concentration ratio $x=c_{\text {TBACl }}^{0} / c_{\text {TBATPB }}^{0}$ ranging from $10^{-4}$ to $10^{2}$. On the other hand, the model $\mathrm{ClO}_{4}{ }^{-}$ion-selective electrode is expected to show such behavior in a rather limited range of $x=$ $\mathrm{C}_{\mathrm{LiClO}}^{0} / \mathrm{C}_{\mathrm{TBAClO}} \approx \mathrm{C}_{4} \approx 10^{2}-10^{5}$, which can be increased a little by increasing the volume ratio $r$, cf. Figure 3 . These results can be of analytical relevance; for example, the decline from the Nernstian behavior at low target ion concentrations can complicate the determination of the ion selectivity coefficients for an ISE based on the Nikolsky equation. ${ }^{1}$

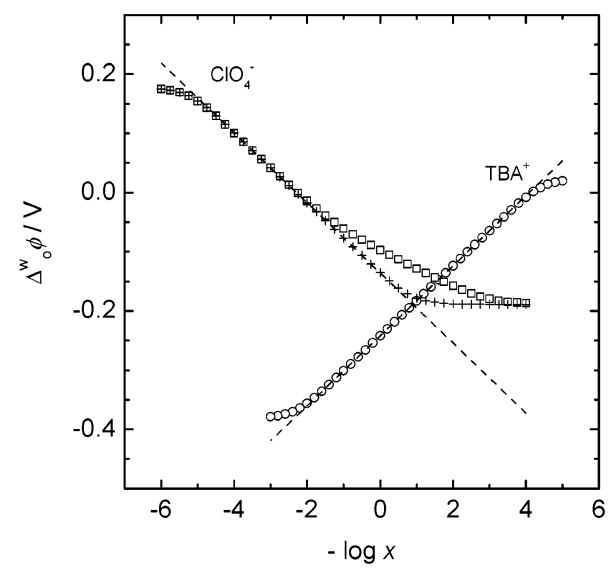

Figure 3. Equilibrium potential difference $\Delta_{\circ}^{\mathrm{w}} \phi$ for the TBACI|TBATPB (O) and $\mathrm{LiClO}_{4} \mid \mathrm{TBAClO}_{4}(\square,+)$ interface vs the logarithm of the concentration ratio $x=c_{\mathrm{TBACl}}^{0} / c_{\mathrm{TBATPB}}^{0}$ and $x=c_{\mathrm{LiClO}_{4}}^{0} /$ $C_{\mathrm{TBACIO}_{4}}^{0}$, respectively, calculated by using eq 10 for the phase volume ratio $r=10^{-3}(O, \square)$ or $1(+)$, and the values of the standard ion transfer potentials taken from Table 1 (ref 12). Lines correspond to the ideal Nernstian behavior.

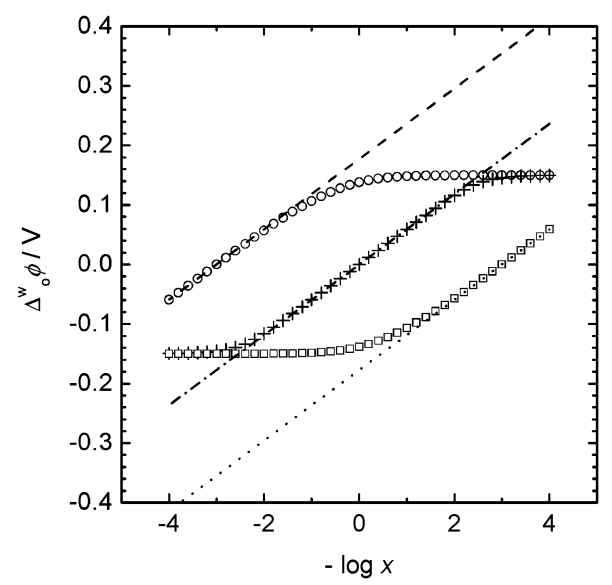

Figure 4. Equilibrium potential difference $\Delta_{\circ}^{\mathrm{w}} \phi$ for the ITIES model of the extraction system vs the logarithm of the concentration ratio $x$ $=c_{\mathrm{IA}}^{0} / c_{\mathrm{IX}}^{0}$ calculated by using eq 11 for the phase volume ratio $r=$ $10^{-3}(O), 1(+)$, and $10^{3}(\square)$, and the values of the standard ion transfer potentials $\Delta_{\mathrm{o}}^{\mathrm{w}} \phi_{1^{+}}^{0}=0, \Delta_{\mathrm{o}}^{\mathrm{w}} \phi_{\mathrm{A}^{-}}^{0}=-0.3 \mathrm{~V}$, and $\Delta_{\mathrm{o}}^{\mathrm{w}} \phi_{\mathrm{X}^{-}}^{0}=0.3 \mathrm{~V}$. Lines corresponds to the ideal Nernstian behavior according to eq 18.

The effect of the phase volume ratio on the equilibrium potential difference for the ITIES model of the extraction system is considerably more pronounced. Figure 4 shows the results of calculations for the same values of the parameters $r$ and $\Delta_{0}^{\mathrm{w}} \phi_{\mathrm{i}}^{0}$ as in Figure 1, but for the initial electrolyte concentrations described by eq 3 . While these plots have a similar shape as those in Figures 1 and 2 , they are shifted by $\log r$ along the $\log x$ axis.

\section{CONCLUSIONS}

Two-phase liquid system IA(w) |IX(0) comprising the interface between the aqueous solution ( $w$ ) of uni-univalent electrolyte IA and an organic solvent solution ( 0 ) of a uni-univalent electrolyte IX with the common cation $\mathrm{I}^{+}$can serve as a simple model of a liquid-membrane ion-selective electrode (ISE) for the cation $\mathrm{I}^{+}$. In contrast to the extraction system, where both electrolytes are initially present in the aqueous phase, the effect of the phase volume ratio on the equilibrium potential difference in the ISE 
model is rather weak, unless the counterions $\mathrm{X}^{-}$and $\mathrm{A}^{-}$differ little in their lipophilicity from the target ion $\mathrm{I}^{+}$. Depending on the lipophilicity of the counterions, both the ISE and extraction model exhibits the Nernstian behavior only in a limited range of the ratio of the initial concentrations of the electrolytes IA and $I X$, which in the ISE model represents the ratio of the initial concentrations of the ion $\mathrm{I}^{+}$in the aqueous and the organic phase, respectively. When this ratio is extremely large or small, the equilibrium potential difference approaches the limiting value that is given by the distribution potential of the excess electrolyte IA or IX, respectively. Similar conclusions can be drawn for the twophase liquid system $\mathrm{AI}(\mathrm{w}) \mid \mathrm{XI}(0)$ with the common anion $\mathrm{I}^{-}$. These results can be of analytical relevance; for example, the decline from the Nernstian behavior can complicate the determination of the ion selectivity coefficient. Qualitatively, the N ernst behavior can be observed in the range of the potential differences $\Delta_{0}^{\mathrm{w}} \phi$ that fulfill the condition $\Delta_{0}^{\mathrm{w}} \phi_{\mathrm{A}^{-}}^{0} \ll \Delta_{0}^{\mathrm{w}} \phi \ll \Delta_{0}^{\mathrm{w}} \phi_{\mathrm{X}^{-}}^{0}$ or $\Delta_{0}^{\mathrm{w}} \phi_{\mathrm{X}^{+}}^{0} \ll \Delta_{0}^{\mathrm{w}} \phi$ $\ll \Delta_{0}^{\mathrm{w}} \phi_{A+}^{0}$. Hence, in designing the ISE for a specific target ion, careful attention should be paid to the selection of the counterions for both the aqueous and the ISE membrane phase.

\section{ACKNOWLEDGMENT}

Z.S. gratefully acknowledges a Visiting Fellowship from the EPFL and the financial support from M inistry of Education, Youth and Sports of the Czech Republic (Grant No. KONTAKT/ME 502). The authors thank Professor Takashi Kakiuchi (University of Kyoto) for helpful comments.

Received for review February 17, 2004. Accepted April 23, 2004.

AC0497297 